\title{
Prevalence of c.1559delT in ALPL, a common mutation resulting in the perinatal (lethal) form of hypophosphatasia in Japanese and effects of the mutation on heterozygous carriers
}

\author{
Atsushi Watanabe ${ }^{1,2}$, Tatsuki Karasugi ${ }^{3}$, Hideaki Sawai ${ }^{4}$, Banyar Than Naing ${ }^{2}$, Shiro Ikegawa ${ }^{3}$, Hideo Orimo ${ }^{2}$ \\ and Takashi Shimada ${ }^{1,2}$
}

Hypophosphatasia (HPP) is an inherited disorder caused by mutations in ALPL that encodes an isozyme of alkaline phosphatase (ALP), TNSALP. One of the most frequent ALPL mutations is c.1559delT, which causes the most severe HPP, the perinatal (lethal) form (pl-HPP). c.1559delT has been found only in Japanese and its prevalence is suspected to be high; however, the allele frequency of c.1559delT in Japanese remains unknown. We designed a screening system for the mutation based on high-resolution melting curve analysis, and examined the frequency of c.1559delT. We found that the c.1559delT carrier frequency is $1 / 480$ (95\% confidence interval, 1/1562-1/284). This indicates that $\sim 1$ in 900000 individuals to have pl-HPP caused by a homozygous c.1559delT mutation. In our analysis, the majority of c.1559delT carriers had normal values of HPP biochemical markers, such as serum ALP and urine phosphoethanolamine. Our results indicate that the only way to reliably detect whether individuals are pl-HPP carriers is to perform the ALPL mutation analysis.

Journal of Human Genetics (2011) 56, 166-168; doi:10.1038/jhg.2010.161; published online 23 December 2010

Keywords: ALPL; c.1559delT; perinatal form of hypophosphatasia; serum alkaline phosphatase; skeletal dysplasia; urine phosphoethanolamine

\section{INTRODUCTION}

Hypophosphatasia (HPP) is an inherited disorder characterized by defective mineralization of the bone and low activity of alkaline phosphatase (ALP; EC 3.1.3.1). ${ }^{1,2}$ HPP is a clinically heterogeneous disease and classified into five forms according to severity and age of onset: perinatal (lethal), infantile (OMIM 241500), childhood (OMIM 241510), adult (OMIM 146300) and odontohypophosphatasia. ${ }^{1}$ All forms of HPP display reduced activity of unfractionated serum ALP and the presence of either one or two pathologic mutations in ALPL, the gene encoding an ALP isozyme (TNSALP).

The perinatal (lethal) form of HPP (pl-HPP) is the most severe HPP with an autosomal recessive mode of inheritance. pl-HPP is more common in Japan than in other countries. ${ }^{3}$ Parents of pl-HPP are heterozygous carriers of ALPL mutations. They show no clinical symptoms, but have reduced serum ALP activity and increased urinary phosphoethanolamine (PEA).$^{4-8}$

$A L P L$ is the only gene known to be associated with HPP. ${ }^{1}$ More than 200 ALPL mutations have been described, accounting for most phenotype variabilities. ${ }^{9}$ HPP is frequently caused by p.E191K and
p.D378V in Caucasians, ${ }^{1}$ whereas p.F327L ${ }^{10}$ and c.1559delT ${ }^{10,11}$ are more common in Japanese. ${ }^{1}$ To date, c.1559delT has only been found in Japanese. ${ }^{11}$ Some patients with pl-HPP are homozygous for c.1559delT, with parents who are heterozygous carriers for the mutation but with no evidence of consanguinity. ${ }^{12,13}$

To identify c.1559delT genotype and to examine its frequency in Japanese, we designed a screening system based on a high-resolution melting curve analysis. ${ }^{14}$ In addition, we examined serum ALP activity and urine PEA in heterozygous c.1559delT carriers to determine whether these markers can identify the HPP carriers.

\section{MATERIALS AND METHODS}

This study was approved by the Institutional Genetic Research Ethics Committee at Nippon Medical School and RIKEN, Center for Genomic Medicine. Blood samples were collected under written informed consents from 3844 healthy Japanese without HPP and its related findings confirmed by orthopedic surgeons. Genomic DNA was extracted from peripheral blood leukocytes using standard protocols. The c.1559delT genotype screening was performed by the small amplicon genotyping method based on high-resolution melting curve

${ }^{1}$ Division of Clinical Genetics, Nippon Medical School Hospital, Tokyo, Japan; ${ }^{2}$ Department of Biochemistry and Molecular Biology, Nippon Medical School, Tokyo, Japan; ${ }^{3}$ Laboratory for Bone and Joint Diseases, Center for Genomic Medicine, RIKEN, Tokyo, Japan and ${ }^{4}$ Department of Obstetrics and Gynecology, Hyogo College of Medicine, Nishinomiya, Japan

Correspondence: Dr A Watanabe, Division of Clinical Genetics, Nippon Medical School Hospital, 1-1-5 Sendagi Bunkyo-ku, Tokyo 113-8603, Japan.

E-mail: aw3703@nms.ac.jp

Received 17 October 2010; revised 23 November 2010; accepted 24 November 2010; published online 23 December 2010 
analysis. ${ }^{14}$ PCR primers for c.1559delT were designed to flank the mutation leaving only single base, including the mutation between the primers: $5^{\prime}$-TT TAAATTCTCGCGCTGGCCCTCTACCCC- $3^{\prime}$ (forward) and $5^{\prime}$-TTTAAATTCC CTCAGAACAGGACGCTC-3' (reverse). PCR conditions were as follows: initial denaturation at $95^{\circ} \mathrm{C}$ for $2 \mathrm{~min}$, followed by 45 cycles at $94^{\circ} \mathrm{C}$ for $30 \mathrm{~s}$ and annealing at $67^{\circ} \mathrm{C}$ for $30 \mathrm{~s}$. After PCR, high-resolution melting was performed in a 96-well plate LightScanner (Idaho Technology, Salt Lake City, UT, USA), which collected data from $55^{\circ} \mathrm{C}$ to $97^{\circ} \mathrm{C}$ at a ramp rate of $0.10^{\circ} \mathrm{C} \mathrm{sec}-1$. The observed number of c.1559delT carriers was divided by the total number of individuals tested to determine the carrier frequency. Serum ALP activity and urine PEA were measured in c.1559delT-heterozygous parents of pl-HPP patients.

\section{RESULTS}

Three ALPL c.1559delT genotypes (wt/wt, wt/c.1559delT and c.1559delT/c.1559delT) were distinguished by the modified small amplicon genotyping method (Figure 1). A heterogeneous c.1559delT mutation (wt/c.1559delT) was detected in 8 of 3844 healthy Japanese subjects, indicating a carrier frequency of $1 / 480$ in the Japanese population (95\% confidence interval, 1/1562-1/284).

The numerical value of ALP activity and urinary PEA varied in heterozygous c.1559delT carriers in parents of perinatal HPP patients. The majority of heterozygous c.1559delT carriers had normal levels of both ALP activity (five out of six males and three out of four females) and urinary PEA (three out of six males and four out of five females) (Figure 2).

\section{DISCUSSION}

Based on our results, we estimated the frequency of c.1559delThomozygous individuals (for example, those with pl-HPP) to be 1/900 000. Previous studies showed that all Japanese pl-HPP patients carried the c.1559delT mutation in at least one allele; half (10/20) were homozygous for c.1559delT and half (10/20) were compound heterozygous for c.1559delT, ${ }^{9-13,15}$ which gives a pl-HPP prevalence of $1 / 450000$ for patients that are homozygous or compound heterozygous for c.1559delT mutation. The other common mutation on ALPL in Japan, p.F327L, is a mild allele whose product retained $\sim 70 \%$ of its enzymatic activity. Patients compound heterozygous for c.1559delT and p.F327L are not associated with pl-HPP. ${ }^{10}$

Biochemical markers, serum ALP activity and urinary PEA levels fell within their normal ranges in the majority of the c.1559delT carriers examined in this paper, whereas heterozygous carriers of the severe forms in other $A L P L$ mutations were reported to have reduced serum ALP activity and increased urinary PEA.4-8 Some possible reasons why c.1559delT carriers display normal marker levels are as follows: the first is the protein properties caused by the different mutation positions. The c.1559delT mutation causes a frameshift downstream of codon L503, resulting in the elimination of the termination codon at 508 and the addition of 80 amino acids at the C-terminus. The mutant protein forms an aggregate that is polyubiquitinated and then degraded in the proteasome. However, the aggregates possess enzyme activity, and may, therefore, influence physiological processes before their destruction. ${ }^{16}$ Second, serum ALP activity is affected by some other factors. The genetic modifier of ALP is reported to have a potential influence on serum ALP activity. ${ }^{17}$ Total ALP value is also elevated by some environmental factors, in vitamin $\mathrm{D}$ deficiency $^{2}$ or in the third trimester of gestation by the increasing placental ALP, which is not affected by TNSALP. ${ }^{18}$ Recently, it was shown that patients who are homozygous for the c.1559delT mutation differed in the severity of HPP, including both their symptoms and serum ALP activity. ${ }^{15}$
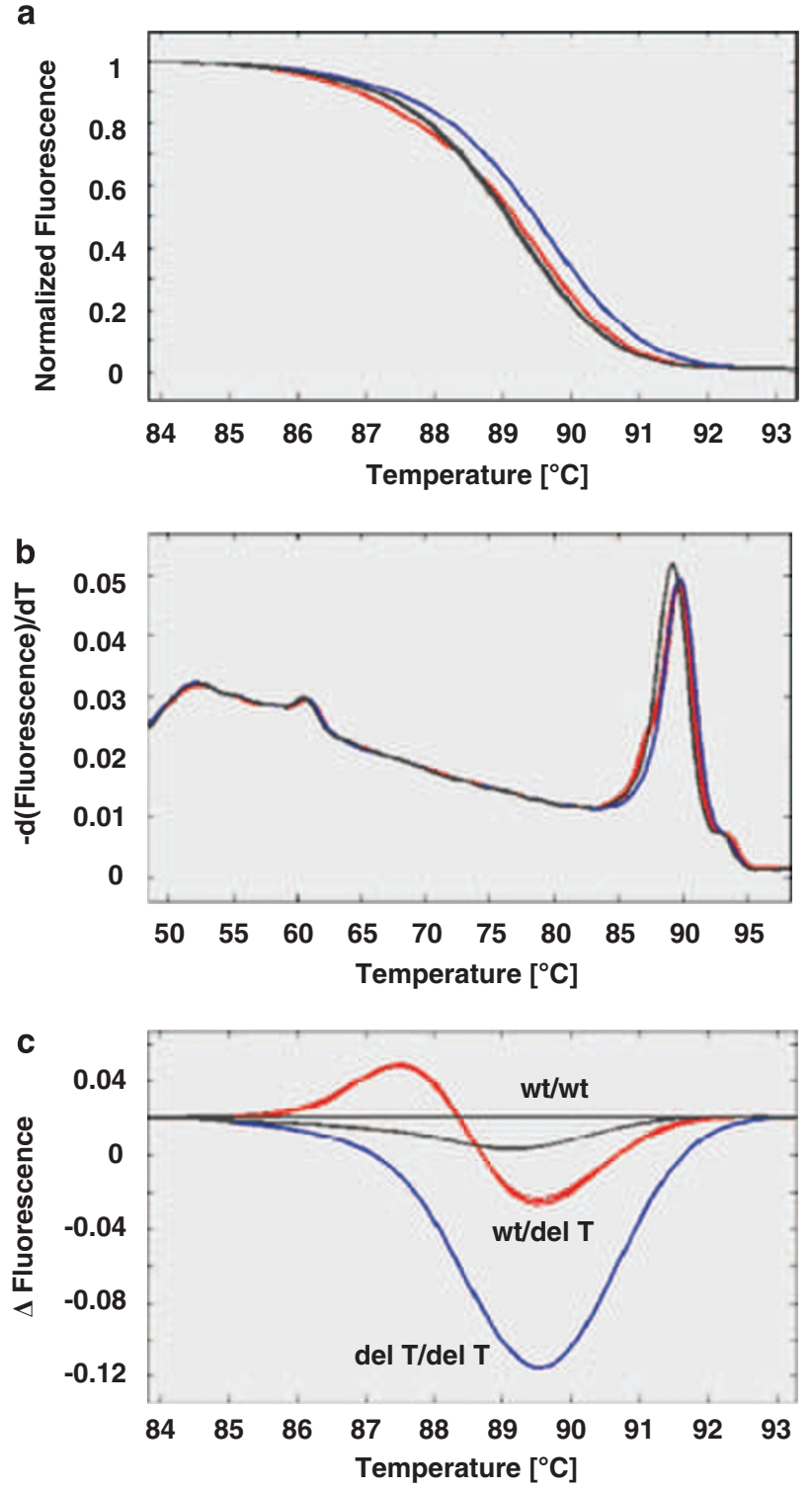

Figure 1 Identification of c.1559delT mutation in ALPL by small amplicon genotyping (SAG) method. (a) Normalized fluorescence plots. (b) $\mathrm{d}$ (fluorescence)/dT plot. (c) The corresponding fluorescence difference plots. Wild-type (wt/wt) samples are in gray; samples heterozygous for c.1559delT (wt/c.1559delT) are in red; and samples homozygous for c.1559delT (c.1559delT/c.1559delT) are in blue. The three genotypes were clearly distinguishable in the SAG method.
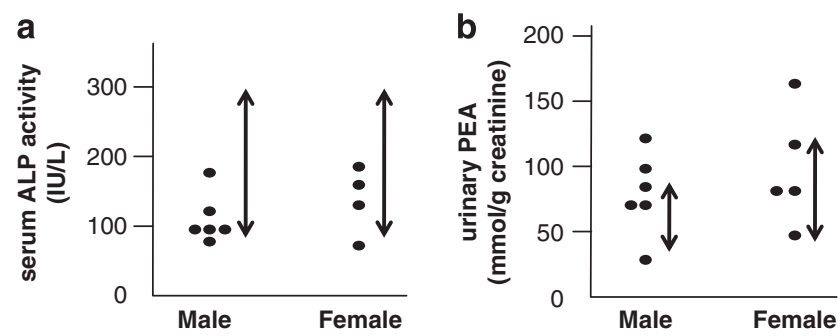

Figure 2 Biochemical marker levels in heterozygous carriers of the ALPL c.1559delT mutation. The serum ALP activity (a) and urinary PEA (b) levels in the majority of heterozygous carriers (wt/c.1559delT) fell within normal ranges (indicated by arrows). 
Thus, the only way to reliably detect the pl-HPP carriers is to perform the $A L P L$ mutation analysis. The small amplicon genotyping method in this study using the high-resolution melting curve analysis is a one-step, single-tube method for detection of specific mutations and faster, simpler and less expensive than the approaches requiring separations or labeled probes. ${ }^{19}$

The screening for c.1559delT in ALPL may be useful for diagnosis of pl-HPP in Japanese to provide optimum genetic counseling for fetal skeletal dysplasia. pl-HPP occasionally could not be diagnosed with sonographic examination in the first trimester because incomplete ossification is an usual finding at this stage of development. ${ }^{20}$ To diagnose pl-HPP, collaborations between obstetricians and clinical geneticists are important and could provide support for parents of prenatal patients suspected of having skeletal dysplasia.

\section{CONFLICT OF INTEREST}

The authors declare no conflict of interest.

\section{ACKNOWLEDGEMENTS}

We wish to thank all the patients and family members who participated in this study, and all the clinicians for referring the families. We thank Hitomi Kondo for technical assistance. This work was supported by a Grant-in-Aid for Research on intractable disease from the Ministry of Health, Labour and Welfare of Japan (Project no. 095/2010).

1 Mornet, E. Hypophosphatasia. Best Pract. Res. Clin. Rheumatol. 22, 113-127 (2008).

2 Whyte, M. P. Hypophosphatasia and the role of alkaline phosphatase in skeletal mineralization. Endocr. Rev. 15, 439-461 (1994).

3 Satoh, N., Murotsuki, A. \& Sawai, H. The birth prevalence rates for skeletal dysplasia in the registration system of the Japan Forum of Fetal Skeletal Dysplasia. J. Jan. Perinat. Neonat. Med. 45, 1005-1007 (2009) (Japanese).

4 Gehring, B., Mornet, E., Plath, H., Hansmann, M., Bartmann, P. \& Brenner, R. E. Perinatal hypophosphatasia: diagnosis and detection of heterozygote carriers within the family. Clin. Genet. 56, 313-317 (1999).

5 Spentchian, M., Merrien, Y., Herasse, M., Dobbie, Z., Gläser, D., Holder, S. E. et al. Severe hypophosphatasia: characterization of fifteen novel mutations in the ALPL gene. Hum. Mutat. 22, 105-106 (2003).

6 Zankl, A., Mornet, E. \& Wong, S. Specific ultrasonographic features of perinatal lethal hypophosphatasia. Am. J. Med. Genet. 146A, 1200-1204 (2008).
7 Sergi, C., Mornet, E., Troeger, J. \& Voigtlaender, T. Perinatal hypophosphatasia: radiology, pathology and molecular biology studies in a family harboring a splicing mutation $(648+1 \mathrm{~A})$ and a novel missense mutation (N400S) in the tissue-nonspecific alkaline phosphatase (TNSALP) gene. Am. J. Med. Genet. 103, 235-240 (2001)

8 Mornet, E., Taillandier, A., Peyramaure, S., Kaper, F., Muller, F., Brenner, R. et al. Identification of fifteen novel mutations in the tissue-nonspecific alkaline phosphatase (TNSALP) gene in European patients with severe hypophosphatasia. Eur. J. Hum. Genet. 6, 308-314 (1998).

9 The Tissue Nonspecific Alkaline Phosphatase Gene Mutations Database. http://www.sesep.uvsq.fr/03_hypo_mutations.php.

10 Michigami, T., Uchihashi, T., Suzuki, A., Tachikawa, K., Nakajima, S. \& Ozono, K. Common mutations F310L and T1559del in the tissue-nonspecific alkaline phosphatase gene are related to distinct phenotypes in Japanese patients with hypophosphatasia. Eur. J. Pediatr. 164, 277-282 (2005).

11 Orimo, H., Goseki-Sone, M., Inoue, M., Tsubakio, Y., Sakiyama, T. \& Shimada, T. Importance of deletion of $\mathrm{T}$ at nucleotide 1559 in the tissue-nonspecific alkaline phosphatase gene in Japanese patients with hypophosphatasia. J. Bone Miner. Metab. 20, 28-33 (2002).

12 Watanabe, A., Yamamasu, S., Shinagawa, T., Suzuki, Y., Takeshita, T., Orimo, H. et al. Prenatal genetic diagnosis of severe perinatal (lethal) hypophosphatasia. J. Nippon Med. Sch. 74, 65-69 (2007).

13 Sawai, H., Kanazawa, N., Tsukahara, Y., Koike, K., Udagawa, H., Koyama, K. et al. Severe perinatal hypophosphatasia due to homozygous deletion of T at nucleotide 1559 in the tissue nonspecific alkaline phosphatase gene. Prenat. Diagn. 23, 743-746 (2003).

14 Gundry, C. N., Dobrowolski, S. F., Martin, Y. R., Robbins, T. C., Nay, L. M., Boyd, N. et al. Base-pair neutral homozygotes can be discriminated by calibrated high-resolution melting of small amplicons. Nucleic Acids Res. 36, 3401-3408 (2008).

15 Nakamura-Utsunomiya, A., Okada, S., Hara, K., Miyagawa, S., Takeda, K., Fukuhara, R. et al. Clinical characteristics of perinatal lethal hypophosphatasia: a report of 6 cases. Clin. Pediatr. Endcrinol. 19, 7-13 (2010).

16 Komaru, K., Ishida, Y., Amaya, Y., Goseki-Sone, M., Orimo, H. \& Oda, K. Novel aggregate formation of a frame-shift mutant protein of tissue-nonspecific alkaline phosphatase is ascribed to three cysteine residues in the C-terminal extension. Retarded secretion and proteasomal degradation. FEBS J. 272, 1704-1717 (2005).

17 Kamatani, Y., Matsuda, K., Okada, Y., Kubo, M., Hosono, N., Daigo, Y. et al. Genomewide association study of hematological and biochemical traits in a Japanese population. Nat. Genet. 42, 210-215 (2010).

18 Whyte, M. P., Landt, M., Ryan, L. M., Mulivor, R. A., Henthorn, P. S., Fedde, K. N. et al. Alkaline phosphatase: placental and tissue-nonspecific isoenzymes hydrolyze phosphoethanolamine, inorganic pyrophosphate, and pyridoxal 5'-phosphate. Substrate accumulation in carriers of hypophosphatasia corrects during pregnancy. J. Clin. Invest. 95, 1440-1445 (1995).

19 Vossen, R. H., Aten, E., Roos, A. \& den Dunnen, J. T. High-resolution melting analysis (HRMA): more than just sequence variant screening. Hum. Mutat. 30, 860-866 (2009).

20 Tongsong, T. \& Pongsatha, S. Early prenatal sonographic diagnosis of congenital hypophosphatasia. Ultrasound Obstet. Gynecol. 15, 252-255 (2000). 